\title{
Enhanced electrical conductivity in graphene-filled polycarbonate nanocomposites by microcellular foaming with sc-CO
}

\author{
Gabriel Gedler, Marcelo Antunes, José Ignacio Velasco* \\ Centre Català del Plàstic, Departament de Ciència dels Materials i Enginyeria \\ Metal·lúrgica, Universitat Politècnica de Catalunya, UPC·BarcelonaTech, \\ Colom 114, E-08222, Terrassa, Spain. \\ *Corresponding author: jose.ignacio.velasco@upc.edu
}

\begin{abstract}
Electrically conductive polycarbonate foams containing a low concentration of graphene nanoplatelets $(0.5 \mathrm{wt} . \%)$ were produced with variable range of expansion ratio by applying a high pressure batch foaming process using sc-CO2. The structure of the foams was assessed by means of SEM, AFM and WAXS, and the electrical conductivity was measured in the foam growing direction. Results showed that electrical conductivity of PC composite foams remarkably increased when compared to that of non-foamed PC composite, with both the electrical conductivity and the main cell size of the foams being directly affected by the resultant expansion ratio of the foam. This interesting result could be explained by the development of an interconnected graphene nanoparticle network composed by increasingly well-dispersed and reoriented graphene nanoplatelets, which was developed into the solid fraction of the foam upon foaming by sudden depressurizing of the plasticized CO2-saturated polycarbonate preform. Some evidences of morphological changes in the graphene nanoplatelets after foaming were obtained by analysing variations in graphene's (002) diffraction plane, whose intensity decreased with foaming. A reduction of the average number of layers in the graphene nanoplatelets was also measured, both
\end{abstract}


evidences indicating that improved dispersion of graphene nanoparticles existed in the PC composite foams. As a result, foams with a proper combination of low density and enhanced electrical conductivity could be produced, enabling them to be used in applications such as EMI shielding.

Keywords: Foams; polycarbonate; electrical conductivity; graphene; carbon dioxide

\section{Introduction}

Graphene-filled polymer composites have been proposed for a wide variety of applications in electronics, photonics and optoelectronics [1-5]. Particularly, polycarbonate (PC) composites containing graphene have been suggested for applications that require the combination of electrical conduction and good barrier properties [6]. Lowering the density of these composites while improving their conductive properties could extend their use to applications where the combination of electrical conduction and lightness is an important asset, as it is the case of electromagnetic interference (EMI) shielding, electrostatic discharge (ESD), electrostatic painting or sensing, among others [7-9]. The combination of both characteristics is also crucial in many aerospace applications, including lightning strike dissipation and electrical charge mitigations in space vehicles [10].

Enhancing conductive filler dispersion and as a consequence lowering its threshold for electrical conduction is one of the most important challenges for the industrial implementation of electrically conductive composites [11]. In this sense, Clingerman et al. [12] have reported evidences of filler dispersion and morphology dependence when measuring the electrical conductivity of PC composites, showing percolation threshold values for graphite-filled and carbon fiber-filled PC composites of 0.11 and 0.9 vol.\%, 
respectively. Pötschke et al. [13] have reported an electrical percolation threshold above 0.5 vol.\% of multiwall carbon nanotubes (MWNT) for MWNT-filled PC composites with an electrical conductivity value of $10^{-9} \mathrm{~S} / \mathrm{cm}$. Sung et al. [14] have found a similar percolation threshold and electrical conductivity for MWNT-filled PC composites, concluding that the degree of aggregation and aspect ratio of the nanotubes crucially affected the structure of the conductive network. Kim and Macosko [6] have recently reported an electrical conductivity of $10^{-9} \mathrm{~S} / \mathrm{m}$ for PC composites containing approximately 1 vol.\% of graphene prepared by means of conventional melt-compounding, while Yoonessi and Gaier [10] reported electrical conductivities that were two orders of magnitude higher $\left(10^{-7} \mathrm{~S} / \mathrm{m}\right)$ for graphene-filled PC composites with 0.14 vol.\% of graphene prepared by the emulsion method. Improved dispersion of the graphene particles, promoted by the emulsion preparation method, was the cause behind this higher electrical conductivity.

We have recently shown that physical foaming using supercritical carbon dioxide may further enhance the electrical conductivity of composites containing conductive carbonbased nanofillers while lowering their density [15], explained on the basis of an improved dispersion of the nanofillers and preferential orientation within the cell walls during foaming, forming a more efficient interconnected conductive network in the material [9, 15-16]. Additionally, we have proven that improved graphene dispersion promotes the formation of partially crystalline PC foams with improved thermal stabilities [17-18], further extending their applicability range.

In this sense, in the present work graphene-filled PC foams were prepared by a process that consisted in the combination of supercritical carbon dioxide foaming and later pressing by compression-molding. Morphological changes of graphene were observed and analyzed by 
X-ray diffraction (XRD) using as a reference graphene's (002) diffraction signal [19-22], and complemented with measurements by atomic force microscopy (AFM), which was used to estimate the thickness of the graphene stacks within the foams. The electrical conductivity of the foams prior and after pressing was determined and related to the improved dispersion and partial exfoliation of the graphene particles promoted by foaming and pressing. This new process opens up the possibility of preparing lightweight conductive composites by means of achieving an effective in-situ dispersion and partial exfoliation of graphene within the composite, hence providing a viable strategy to extend the range of applications of graphene-filled PC foams.

\section{Experimental procedure}

\section{Materials and composite preparation}

A commercial bisphenol A polycarbonate (Lexan 123R) supplied by Sabic (Sittard, Ned.), with a density of $1.2 \mathrm{~g} / \mathrm{cm}^{3}$ and a melt flow index (MFI) of $17.5 \mathrm{dg} / \mathrm{min}$, measured at 300 ${ }^{\circ} \mathrm{C}$ and $1.2 \mathrm{~kg}$ according to ISO 1133 , was used. Graphene nanoplatelets (GnP) were supplied by XG Sciences, Inc. (MI, USA) and had a thickness of 6 to $8 \mathrm{~nm}$, an average platelet diameter of $15 \mu \mathrm{m}$ and a bulk density of $2.2 \mathrm{~g} / \mathrm{cm}^{3}$, as reported by the manufacturer.

Composite samples (GnP-PC) were prepared by initially melt compounding the PC with 0.5 wt\% graphene nanoplatelets $(0.25$ vol.\%) using a Brabender Plasti-Corder internal mixer at $180{ }^{\circ} \mathrm{C}$ during 6 min. The composite was then transferred into a circular-shaped mould having a nominal diameter of $74 \mathrm{~mm}$ and a thickness of $3.5 \mathrm{~mm}$ and compressionmolded using a hot-plate press (IQAP LAP PL-15) at a temperature of $220{ }^{\circ} \mathrm{C}$ and a pressure of 45 bar during 2 minutes [17]. 


\section{$\mathrm{CO}_{2}$ dissolution and foaming}

The preparation of the foams consisted in the dissolution of supercritical carbon dioxide in the foaming precursors obtained after compression-molding in the hot-plate press and subsequent expansion. Initially, supercritical carbon dioxide $\left(\mathrm{sCO}_{2}\right)$ was dissolved into the precursors inside a high pressure vessel at temperatures between 200 and $213{ }^{\circ} \mathrm{C}$, with pressures that varied between 120 and 160 bar during dissolution times up to $160 \mathrm{~min}$. This dissolution time was defined as the time period required to heat each sample from room temperature to the dissolution temperature $\left(200-213^{\circ} \mathrm{C}\right)$ plus the time the system was kept under pressure at said dissolution temperature. These parameters were selected after previous optimization of the temperature, pressure and time ranges required for foaming these materials. Foaming took place by applying a sudden pressure drop of $4 \mathrm{bar} / \mathrm{s}$ from the selected pressure until a residual pressure that ranged between 0 and 20 bar (consult Table 1 for further details). Cellular composite samples were coded by the letter "F" followed by the value of their relative density, estimated by dividing the density of each sample by the density of the foaming precursor. Density values were measured according to standard ISO 845.

A photograph of the unfoamed GnP-PC composite used as foaming precursor and a photograph of a characteristic GnP-PC foam are shown side by side in Figure 1.

In order to analyze the effect of the deformation of the cellular structure of the foam on the values of electrical conductivity, an additional pressing stage, which consisted in compressing the foams until a given thickness deformation, was applied (consult S1 and S2 for further information). 


\section{Morphology and microstructure}

A JEOL JSM-5610 scanning electron microscope working with $15 \mathrm{kV}$ and a working distance of $30 \mathrm{~mm}$ was used to measure the average cell size $(\phi)$ of the cellular composites [23]. Samples were previously prepared by brittle fracturing after immersion in liquid nitrogen and subsequently depositing a thin layer of gold at their surface using a BAL-TEC SCD005 Sputter Coater in argon atmosphere.

In order to estimate the average thickness of the graphene nanoplatelets in the composite and foams and as a result the number of individual graphene layers forming them, AFM in the tapping height mode signal was used. Measurements were carried out using a Multimode 8 AFM head attached to a Nanoscope V electronics (Bruker) using a silicon single beam cantilever probe with a silicon oxide tip with a nominal spring constant of 0.35 $\mathrm{nN} / \mathrm{nm}$ and a vertical resolution of $0.2 \mathrm{~nm}$. AFM samples were prepared by cryogenically fracturing the several composites and subsequently cutting $10 \mathrm{~mm} \times 10 \mathrm{~mm}$ squares leaving the fracture surface untouched for the measurement.

The unfoamed PC-graphene composite and resulting foams were analyzed by X-ray diffraction (XRD) using a Panalytical diffractometer. CuK $\alpha$ radiation $(\lambda=1.54 \AA)$ was used, with the diffractometer operating at $40 \mathrm{kV}$ and $40 \mathrm{~mA}$ at room temperature, scanning from 2 to $60^{\circ}$ using a step size of $0.02^{\circ}$.

\section{Electrical conductivity measurements}

The transverse dc conductivity, i.e., the through-plane electrical conductivity, of the composites was measured on samples having a nominal thickness of $0.5 \mathrm{~mm}$. A pA 
meter/dc voltage source HP 4140B with a two-probe set was used. The connections were set up in the electrostatic light-shielded test box HP 16055A using electrolytic copper sheet electrodes. Samples were cut to $20 \mathrm{~mm} \times 20 \mathrm{~mm}$ squares and their thickness was reduced to approximately $1 \mathrm{~mm}$ by polishing using sandpaper. After polishing, a thin silver conductive paint layer with a resistance per area ranging from 0.01 to $0.1 \Omega / \mathrm{cm}^{2}$ was deposited on both top and bottom surfaces of the sample in order to guarantee a good electrical contact. A programmable dc voltage feature with a range of $0-0.05 \mathrm{~V}$ and a voltage step of $0.01 \mathrm{~V}$, a hold time of 5 seconds and a step delay time of 5 seconds, was used. In order to obtain the value of the electrical resistance of the sample $(R)$, a characteristic intensity (I)-Voltage (V) curve was plotted for each sample, with $R$ being calculated as the slope of the I-V curve. The electrical resistivity was determined by simply taking into account the surface area of each sample and their respective thickness. The electrical conductivity was calculated as the reciprocal of the electrical resistivity.

\section{Results and discussion}

\section{Cellular morphology of the foams}

The morphology of GnP-PC foams was determined by the combination of $\mathrm{sCO}_{2}$ dissolution and foaming conditions and the presence of the graphene nanoplatelets, which resulted in foams with different relative densities and average cell sizes that ranged from around 70 to $150 \mu \mathrm{m}$ (see values presented in the last column of Table 1). As can be seen by the micrographs presented in Figure 2, foams displayed a characteristic quasi-homogeneous closed-cell structure. 
In general, for a fixed foaming temperature, foams grew more with increasing saturation pressure, resulting in lower densities and higher average cell sizes (compare the values of density and $\phi$ presented in Table 1 for foams prepared at $205^{\circ} \mathrm{C}$ using different saturation pressures). This can be explained by the fact that nucleation velocity may be assumed to be constant for a given pressure, hence $\phi$ being only affected by the pressure drop, meaning that higher values of the applied pressure drop led to higher values of the average cell size. As expected, foams prepared at higher temperatures presented the highest average cell size values (around $150 \mu \mathrm{m})$ and the lowest values of density $\left(0.39 \mathrm{~g} / \mathrm{cm}^{3}\right.$, i.e., an expansion ratio of almost 3).

\section{Microstructure of the foams}

GnP particles having a range of sizes (surface dimension) could be seen by AFM in both as received as well as on the fracture surfaces of foams (Figures $3 \mathrm{a}$ and $3 \mathrm{~b}$ respectively). AFM under tapping mode was used to estimate the height of graphene stacks on the fracture surface of the unfoamed composite and resulting foams. This value, although not really being the exact value of stack thickness, can be taken as a guiding value and hence can be used to assess the average number of graphene layers. The as-received graphene nanoplatelets $(\mathrm{GnP})$ already showed a very broad thickness range between 30 and $200 \mathrm{~nm}$ with an average thickness around $75 \mathrm{~nm}$, clearly above the value indicated by the manufacturer, leading to an average number of graphene layers above 200, with this value slightly decreasing for GnP-PC composite and considerably reducing for GnP-PC foams.

Particularly, foams showed an average height of graphene stacks of only a few nanometers, with some of the graphene sheets presenting thicknesses as low as $0.5 \mathrm{~nm}$. Taking into 
account that the vertical resolution of the tip used in the measurements as brand new is 0.2 $\mathrm{nm}$, some of these particular measurements can be taken as almost individual graphene layers [24]. So, these results show how the average number of layers in the graphene stacks was significantly reduced from tens of sheets in the original $\mathrm{GnP}$ nanoplatelets and unfoamed composite to only a few in the foams. This fact seems to indicate that $\mathrm{sCO}_{2}$ foaming induced the exfoliation of some graphene stacks, which was attributed to a combination of the presence of $\mathrm{CO}_{2}$ molecules in the interlayer space of the stacks and the high pressure drop used in the process. Also, the high affinity of $\mathrm{CO}_{2}$ molecules for the surface of carbon-based structures has been vastly studied [25-29]. In this sense, taking into account the high surface area of graphene nanoplatelets and the high $\mathrm{CO}_{2}$ pressure used in the process, the interaction between $\mathrm{CO}_{2}$ molecules and the piled up graphene sheets could have increased. It is possible then that $\mathrm{CO}_{2}$ molecules placed between graphene sheets could desorb just at the moment of decompression during foaming, inducing the separation and thus partial exfoliation of graphene stacks. In addition, the presence of PC makes more difficult for the re-stacking of graphene nanoplatelets after their partial exfoliation due to rapid cooling of the matrix during depressurization, globally resulting in composites with well-dispersed and partially exfoliated graphene nanoplatelets.

Although it was possible to establish a tendency in terms of graphene stack thickness reduction with foaming, AFM measurements are still conditioned by the roughness of the analyzed fracture surfaces. That is why it was decided to make a deeper analysis of the average number of graphene layers $(n)$ in the several materials by applying the DebyeScherrer equation to the characteristic (002) graphene peak obtained from XRD measurements: 
$n=\frac{L_{a}}{d_{(002)}}$, where $L_{a}=\frac{0.89 \lambda}{\beta_{(002)} \cos \theta_{(002)}}$

where $L_{a}$ is the stacking height, $d_{(002)}$ is the interplanar distance corresponding to peak (002), $\lambda$ is the wavelength and $\beta_{(002)}$ is the full width at half maximum of peak (002).

\section{Graphene's (002) diffraction peak analysis}

As can be seen in Figure 4, XRD spectra showed the presence of a characteristic graphiticlike structure in the original graphene nanoplatelets $(\mathrm{GnP})$, as assessed by the strong sharp peak observed at $2 \theta=26.5^{\circ}$ corresponding to the (002) crystal diffraction plane of graphite [30]. The unfoamed composite (GnP-PC) showed this same peak but with a considerable intensity reduction when compared to the original $\mathrm{GnP}$, which was related to a combination of a lower graphene concentration and improved dispersion during melt-compounding. Interestingly, the unfoamed composite treated with supercritical carbon dioxide (GnP-PC$\mathrm{CO}_{2}$ ) presented a less intense and broader (002) peak than GnP-PC, demonstrating the effect of $\mathrm{sCO}_{2}$ in improving the dispersion and separation of the graphene layers within the PC matrix. Nevertheless, only with foaming the composite it was possible to see an important reduction in the intensity of the characteristic (002) graphite peak, in some cases almost resulting in its disappearance. The intensity of (002) peak could be reduced with reducing the relative density of foams, i.e., with increasing their volume expansion, indicating that the graphene particles were progressively dispersed and partially exfoliated within the PC matrix with expanding [19-20].

As mentioned, experimental (002) diffraction peak intensity signals were used to calculate the average number of graphene layers $(n)$ according to the Debye-Scherrer equation [21- 
22]. It was found that the average number of layers of the graphene stacks present in the GnP-PC composite was much higher than in foams, where the value was significantly reduced, in some cases to only a few layers (as in the case of F046 cellular composite), once again indicating that some graphene exfoliation took place during expansion. The values of the average number of graphene layers determined using the Debye-Scherrer equation are presented in Table 2 for all composites.

\section{Electrical conductivity}

It is to be expected that an improved dispersion of the graphene nanoplatelets within the PC matrix could promote the creation of a conductive graphene network and as a consequence lead to composites with high electrical conductivities.

As can be seen in Figure 5(a), the electrical conductivity of foams increased with the expansion ratio up to $10^{-8} \mathrm{~S} / \mathrm{cm}$. An improved dispersion of the graphene nanoplatelets within the PC could be behind this increment in electrical conductivity, related to the formation of a more efficient conductive network [31]. Although these values were lower than expected based on the high theoretical electrical conductivity of graphene $\left(10^{7} \mathrm{~S} / \mathrm{m}\right.$ and $10^{2} \mathrm{~S} / \mathrm{m}$ for parallel and perpendicular directions respectively according to the $\mathrm{GnP}$ manufacturer), a network of physically connected graphene nanoplatelets was formed. Still,

for the lower expansion ratios, graphene nanoplatelets did not reach the minimum proximity required for electrical conduction. The possible preferential orientation and enhanced dispersion of graphene nanoplatelets achieved with the expansion can be seen as the cause behind the formation of a more effective electrically-conductive network throughout the material. This is supported by results plotted in Figure 5(b), with the 
electrical conductivity of foams increasing with the average cell size. Nevertheless, there seems to be a threshold approximately at an expansion ratio of 2 above which the electrical conduction mechanism reduces its efficiency, as can be seen by the abrupt slope reduction in both Figs. 5a and 5b. A possible explanation would be that above this expansion ratio and respective cellular growth, the interparticle distance increases reducing the efficiency of the conduction mechanism.

The highest electrical conductivity value found for the composite foams prepared was 1.34 $\times 10^{-8} \mathrm{~S} / \mathrm{cm}$, corresponding to a GnP volume fraction in the whole foam of 0.09 calculated taking into account a foam's density of $0.390 \mathrm{~g} / \mathrm{cm}^{3}$, that is, considering a three-phase system (air, polymer and particles).

These results also support the previous hypothesis based on the improvement of the electrical conductivity of the composites due to a higher proximity between graphene nanoplatelets. In this sense, there was a transition from insulator to semiconductor behaviour observed in some samples with an improvement of 4 orders of magnitude after foaming. This suggests that the enhancement of dispersion and reduction of particle-particle distance to promote the electrical conduction by tunneling is taking place, due to the interactions between $\mathrm{CO}_{2}$ molecules-graphitic structures combined with suddenly depressurization of large pressure differences during foaming. It seems that the expansion rate achieved was beneficial for the better dispersion of particles, however higher expansion ratios could induce a counter effect when the particles could start to separate from each other during large stretching of the solid fraction of foams. 
Figure 6 displays the electrical conductivity of foams as a function of $\mathrm{GnP}$ volume fraction. As can be seen, the electrical conductivity linearly increased with decreasing GnP's volume fraction.

It can be noticed that one of the lowest electrical percolation thresholds reported so far for PC-graphene unfoamed composites has been 0.14 vol.\% graphene, corresponding to a conductivity value of $1.03 \times 10^{-9} \mathrm{~S} / \mathrm{cm}$ [10]. Other graphene-filled polymer composites found in the literature have been reported to display even lower graphene percolation thresholds of around 0.1 vol. $\%$ with electrical conductivities close to $10^{-7} \mathrm{~S} / \mathrm{cm}$ [32]. In contrast to these previously realized works, the composites analyzed here were prepared using industrial-scalable methods such as melt-compounding, compression-molding and physical foaming, with graphene volume fraction down to 0.09, achieving an electrical conductivity of $1.34 \times 10^{-8} \mathrm{~S} / \mathrm{cm}$. The highest electrical conductivity value obtained after foaming considering similar graphene volume fractions is very close to the values reported in the literature for unfoamed PC-graphene composites $[10,32]$ but in our case with a reduction of almost up to $70 \%$ in weight of material making it very attractive for applications in sector such as transport, aerospace and electronics.

\section{Conclusions}

Lightweight graphene particle-filled polycarbonate composites with improved graphene dispersion were successfully prepared using a $\mathrm{sCO}_{2}$ dissolution foaming process. Microstructural and morphological changes were observed in terms of the crystalline structure of graphene stacks present in the foams, with an intensity reduction and in some cases almost disappearance of the characteristic (002) diffraction peak of graphene being 
observed after foaming. AFM measurements of the thickness of graphene stacks supported that separation and partial exfoliation of the particles down to a few graphene layers took place with foaming.

The electrical conductivity of foams increased with decreasing relative density and increasing the average cell size, which was related to a favourable orientation and reduction of the space between graphene nanoplatelets for electrical conduction enhancement, suggesting that the nanoplatelets reduced their separation distance due to the interactions between $\mathrm{CO}_{2}$ molecules-graphitic structures combined with the suddenly depressurization of large pressure differences during foaming. This reduction of the distance required for electrical conduction by tunneling combined with the dispersion/exfoliation of particles and the orientation of molecules after foaming promoted the formation of an improved electrically conductive network for composites at a low graphene volume fraction suggesting the possible use of these materials in piezoelectric, EMI shielding and ESD applications in sectors such as transport and aerospace.

\section{Acknowledgments}

The authors would like to acknowledge the Spanish Ministry of Economy and Competitiveness for the financial support of project MAT2014-56213.

\section{References}

[1] Chen L, Zhang M, Wei W. Graphene-based composites as cathode materials for lithium ion batteries. J Nanomater. 2013; 2013: 1-8.

[2] Bonaccorso F, Sun Z, Hasan T, Ferrari AC. Graphene photonics and optoelectronics. Nat. Photonics. 2010; 4: 611-622. 
[3] Zhang M, Lei D, Yin X, Chen L, Li Q, Wang Y, Wang T. Magnetite/graphene composites: microwave irradiation synthesis and enhanced cycling and rate performances for lithium ion batteries. J Mater. Chem. 2010; 20: 5538-5543.

[4] Paek SM, Yoo E, Honma E. Enhanced cyclic performance and lithium storage capacity of $\mathrm{SnO}_{2} /$ Graphene nanoporous electrodes with three-dimensionally delaminated flexible structure. Nano Lett. 2008; 9: 72-75.

[5] Wu ZS, Ren W, Wen L, Gao L, Zhao J, Chen Z, Zhou G, Li F, Cheng HM. Graphene anchored with $\mathrm{Co}_{3} \mathrm{O}_{4}$ nanoparticles as anode of lithium ion batteries with enhanced reversible capacity and cyclic performance. ACS Nano. 2010; 4: 3187-3194.

[6] Kim H, Macosko CW. Processing-property relationships of polycarbonate/graphene composites. Polymer. 2009; 50: 3797-3809.

[7] Chung DDL. Electrical applications of carbon materials. J Mater. Sci. 2004; 39: 26452661.

[8] Liang J, Wang Y, Huang Y, Ma Y, Liu Z, Cai J, Zhang C, Gao H, Chen Y. Electromagnetic interference shielding of graphene/epoxy composites. Carbon. 2009; 47: 922-925.

[9] Zhang HB, Yan Q, Zheng WG, He Z, Yu ZZ. Tough graphene-polymer microcellular foams for electromagnetic interference shielding. ACS Appl. Mater. Interfaces. 2011; 3: 918-924.

[10] Yoonessi M, Gaier JR. Highly conductive multifunctional graphene polycarbonate nanocomposites. ACS Nano. 2010; 4: 7211-7220.

[11] Hu K, Kulkarni DD, Choi I, Tsukruk VV. Graphene-polymer nanocomposites for structural and functional applications. Prog. Polym. Sci. 2014; 39: 1934-1972. 
[12] Clingerman ML, Weber EH, King JA, Schulz KH. Synergistic effect of carbon fillers in electrically conductive nylon 6,6 and polycarbonate based resins. Polym. Compos. 2002; 23: 911-924.

[13] Pötschke P, Bhattacharyya AR, Janke A. Carbon nanotube-filled polycarbonate composites produced by melt mixing and their use in blends with polyethylene. Carbon. 2004; 42: 965-969.

[14] Sung YT, Han MS, Song KH, Jung JW, Lee HS, Kum CK, Joo J, Kim WN. Rheological and electrical properties of polycarbonate/multi-walled carbon nanotube composites. Polymer. 2006; 47: 4434-4439.

[15] Antunes M, Mudarra M, Velasco JI. Broad-band electrical conductivity of carbon nanofibre-reinforced polypropylene foams. Carbon. 2011; 49: 708-717.

[16] Ameli A, Jung PU, Park CB. Electrical properties and electromagnetic interference shielding effectiveness of polypropylene/carbon fiber composite foams. Carbon. 2013; 60: $379-391$.

[17] Gedler G, Antunes M, Realinho V, Velasco JI. Novel polycarbonate-graphene nanocomposite foams prepared by $\mathrm{CO}_{2}$ dissolution. IOP Conf. Ser.: Mater. Sci. Eng. 2012; 31: 012008.

[18] Gedler G, Antunes M, Realinho V, Velasco JI. Thermal stability of polycarbonategraphene nanocomposite foams. Polym. Degra. Stab. 2012; 97: 1297-1304.

[19] Song P, Cao Z, Cai Y, Zhao L, Fang Z, Fu S. Fabrication of exfoliated graphene-based polypropylene nanocomposites with enhanced mechanical and thermal properties. Polymer. 2011; 52: 4001-4010. 
[20] Liang J, Huang Y, Zhang L, Wang Y, Ma Y, Cuo T, Chen Y. Molecular-level dispersion of graphene into poly(vinyl alcohol) and effective reinforcement of their nanocomposites. Adv. Funct. Mater. 2009; 19: 2297-2302.

[21] Sakintuna B, Yürüm Y, Çetinkaya S. Evolution of carbon microstructures during the pyrolysis of turkish elbistan lignite in the temperature range $700-1000{ }^{\circ} \mathrm{C}$. Energy Fuels. 2004; 18: 883-888.

[22] Rao CNR, Biswas K, Subrahmanyam KS, Govindaraj A. Graphene, the new nanocarbon. J Mater. Chem. 2009; 19: 2457-2469.

[23] Sims GLA, Khunniteekool C. Cell size measurement of polymeric foams. Cell. Polym. 1994; 13: 137-146.

[24] Novoselov KS, Geim AK, Morozov SV, Jiang D, Zhang Y, Dubonos SV, Grigorieva IV, Firsov AA. Electric field effect in atomically thin carbon films. Science. 2004; 306: 666-669.

[25] Alexiadis A, Kassinos S. Molecular dynamic simulations of carbon nanotubes in $\mathrm{CO}_{2}$ atmosphere. Chem. Phys. Lett. 2008; 460: 512-516.

[26] Mishra AK, Ramaprabhu S. Carbon dioxide adsorption in graphene sheets. AIP Adv. 2011; 1: 032152.

[27] Lithoxoos GP, Labropoulos A, Peristeras LD, Kanellopoulos N, Samios J, Economou IG. Adsorption of $\mathrm{N}_{2}, \mathrm{CH}_{4}, \mathrm{CO}$ and $\mathrm{CO}_{2}$ gases in single walled carbon nanotubes: $\mathrm{A}$ combined experimental and Monte Carlo molecular simulation study. J. Supercrit. Fluids. 2010; 55: 510-523.

[28] Montoya A, Mondragón F, Truong TN. $\mathrm{CO}_{2}$ adsorption on carbonaceous surfaces: a combined experimental and theoretical study. Carbon. 2003; 41: 29-39. 
[29] Bienfait M, Zeppenfeld P, Dupont-Pavlovsky N, Muris M, Johnson MR, Wilson T, DePies M, Vilches OE. Thermodynamics and structure of hydrogen, methane, argon, oxygen, and carbon dioxide adsorbed on single-wall carbon nanotube bundles. Phys. Rev. B. 2004; 70: 035410.

[30] Pham TA, Kim JS, Kim JS, Jeong YT. One-step reduction of graphene oxide with 1glutathione. Colloids Surf. A Physicochem. Eng. Asp. 2011; 384: 543-548.

[31] Antunes M, Realinho V, Velasco JI. Foaming behaviour, structure, and properties of polypropylene nanocomposites foams. J Nanomat. 2010; 2010: 1-11.

[32] Stankovich S, Dikin DA, Dommett GHB, Kohlhaas KM, Zimney EJ, Stach EA, Piner

RD, Nguyen ST, Ruoff RS. Graphene-based composite materials. Nature. 2006; 442: 282286. 


\section{Figure captions}

Figure 1. Photographs of the transversal cut of a GnP-PC foaming precursor (left) and a GnP-PC foam (right).

Figure 2. SEM micrographs of (a) F058 and (b) F034 GnP-PC foams.

Figure 3. AFM images of (a) original graphene nanoplatelets $(\mathrm{GnP})$ and (b) graphene particles present in F038 foams. Black arrows indicate some of the observed graphene particles.

Figure 4. Characteristic XRD spectra of the original graphene nanoplatelets (GnP), unfoamed GnP-PC, unfoamed composite treated with $\mathrm{CO}_{2}\left(\mathrm{GnP}-\mathrm{PC}-\mathrm{CO}_{2}\right)$ and $\mathrm{F} 058$ foam. Insert shows a detail of the characteristic (002) crystal diffraction plane of graphite.

Figure 5. Electrical conductivity of GnP-PC foams as a function of (a) expansion ratio and (b) average cell size.

Figure 6. Electrical conductivity of GnP-PC foams as a function of graphene volume fraction. 
Table 1. Experimental conditions used for the preparation of the GnP-PC foams and resulting expansion ratios and average cell sizes $(\phi)$.

\begin{tabular}{|c|c|c|c|c|c|c|}
\hline \multirow[b]{2}{*}{ Code } & \multicolumn{5}{|c|}{ Foaming conditions } & \multirow[b]{2}{*}{$\phi(\mu \mathrm{m})$} \\
\hline & $\begin{array}{c}\text { Temperature } \\
\left({ }^{\circ} \mathrm{C}\right)\end{array}$ & $\begin{array}{c}\text { Pressure } \\
\text { (bar) }\end{array}$ & $\begin{array}{l}\text { Dissolution time } \\
(\min )\end{array}$ & $\begin{array}{l}\text { Density } \\
\left(\mathrm{g} / \mathrm{cm}^{3}\right)\end{array}$ & $\begin{array}{c}\text { Expansion } \\
\text { ratio }\end{array}$ & \\
\hline F078 & 200 & 135 & 60 & 0.90 & 1.3 & $73 \pm 4$ \\
\hline F058 & 210 & 120 & 60 & 0.67 & 1.7 & $86 \pm 4$ \\
\hline F049 & 205 & 140 & 160 & 0.56 & 2.0 & $92 \pm 5$ \\
\hline F046 & 205 & 150 & 80 & 0.53 & 2.1 & $94 \pm 5$ \\
\hline F038 & 205 & 160 & 60 & 0.43 & 2.6 & $97 \pm 5$ \\
\hline F034 & 213 & 160 & 40 & 0.39 & 2.9 & $146 \pm 7$ \\
\hline
\end{tabular}


Table 2. Average number of graphene layers $(n)$ determined using the Debye-Scherrer equation for GnP, GnP-PC composites and GnP-PC foams. The values of the stacking height $\left(L_{a}\right)$ and the experimental interplanar distance of peak $(002)\left(d_{(002)}\right)$ are also included.

\begin{tabular}{cccc}
\hline Code & $L_{a}(\AA)$ & $d_{(002)}(\AA)$ & $n$ \\
\hline GnP & 160 & 3.36 & 50 \\
\hline GnP-PC & 270 & 3.36 & 80 \\
GnP-PC-CO 2 & 100 & 3.35 & 30 \\
\hline F078 & 100 & 3.35 & 30 \\
F058 & 53 & 3.36 & 16 \\
F049 & 89 & 3.36 & 26 \\
F046 & 28 & 3.35 & 8 \\
F038 & 47 & 3.36 & 14 \\
F034 & 32 & 3.35 & 9 \\
\hline
\end{tabular}



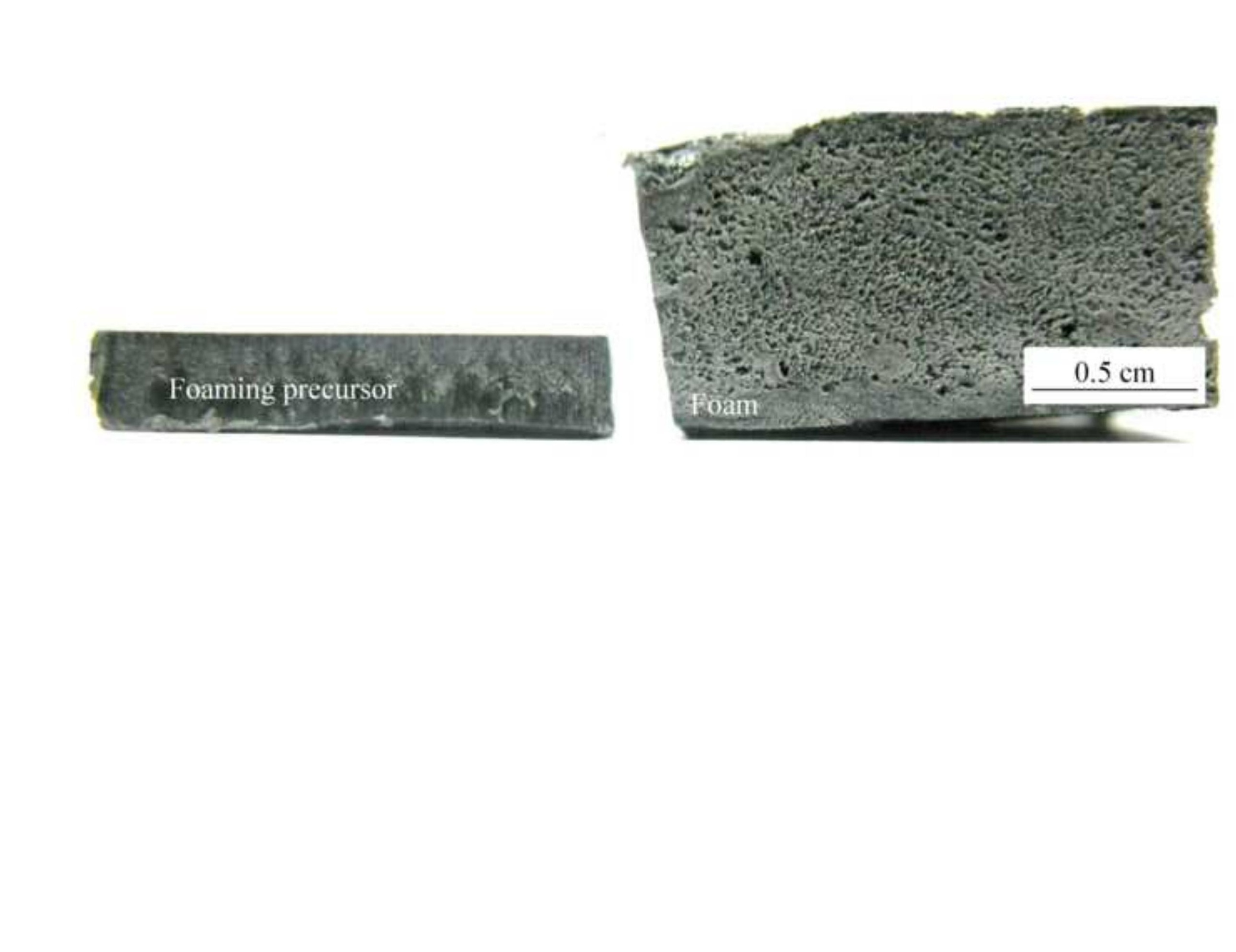
(a) 523,02035050030 Y

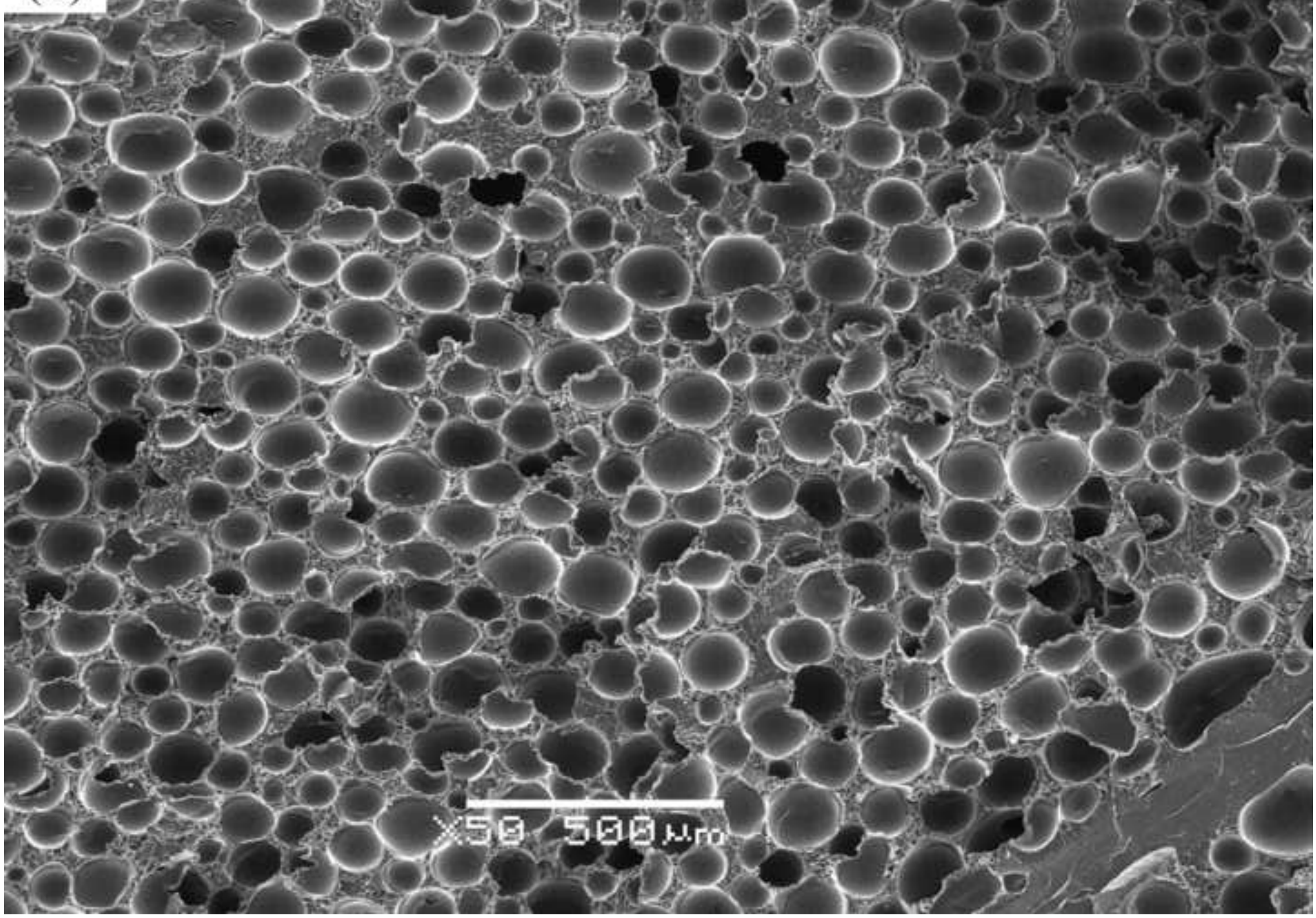







(a)



\section{Click here to download Figure Fig 3 a.tif}


Click here to download Figure Fig 3b.tif $\underline{\underline{\Perp}}$

(b)

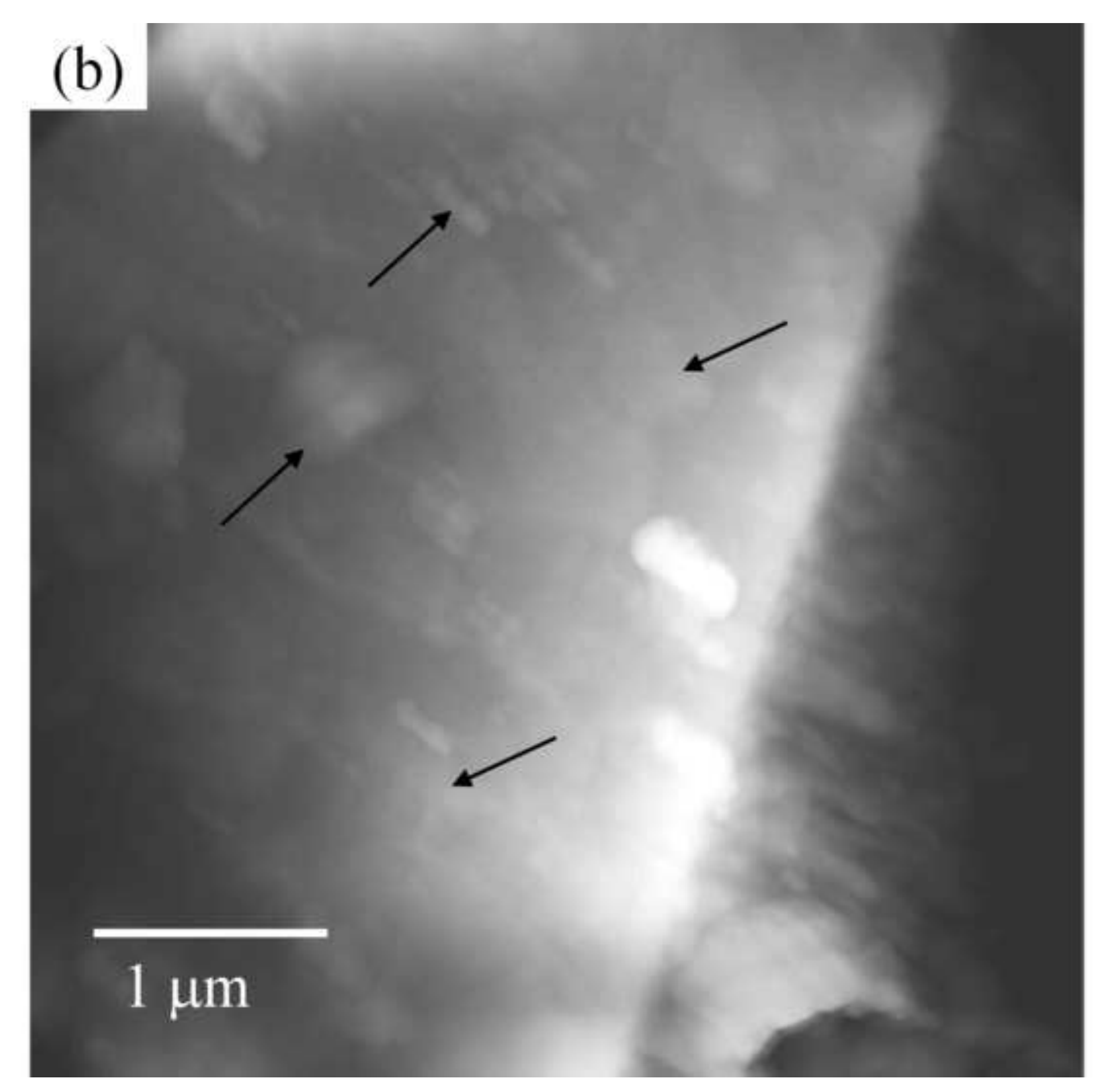




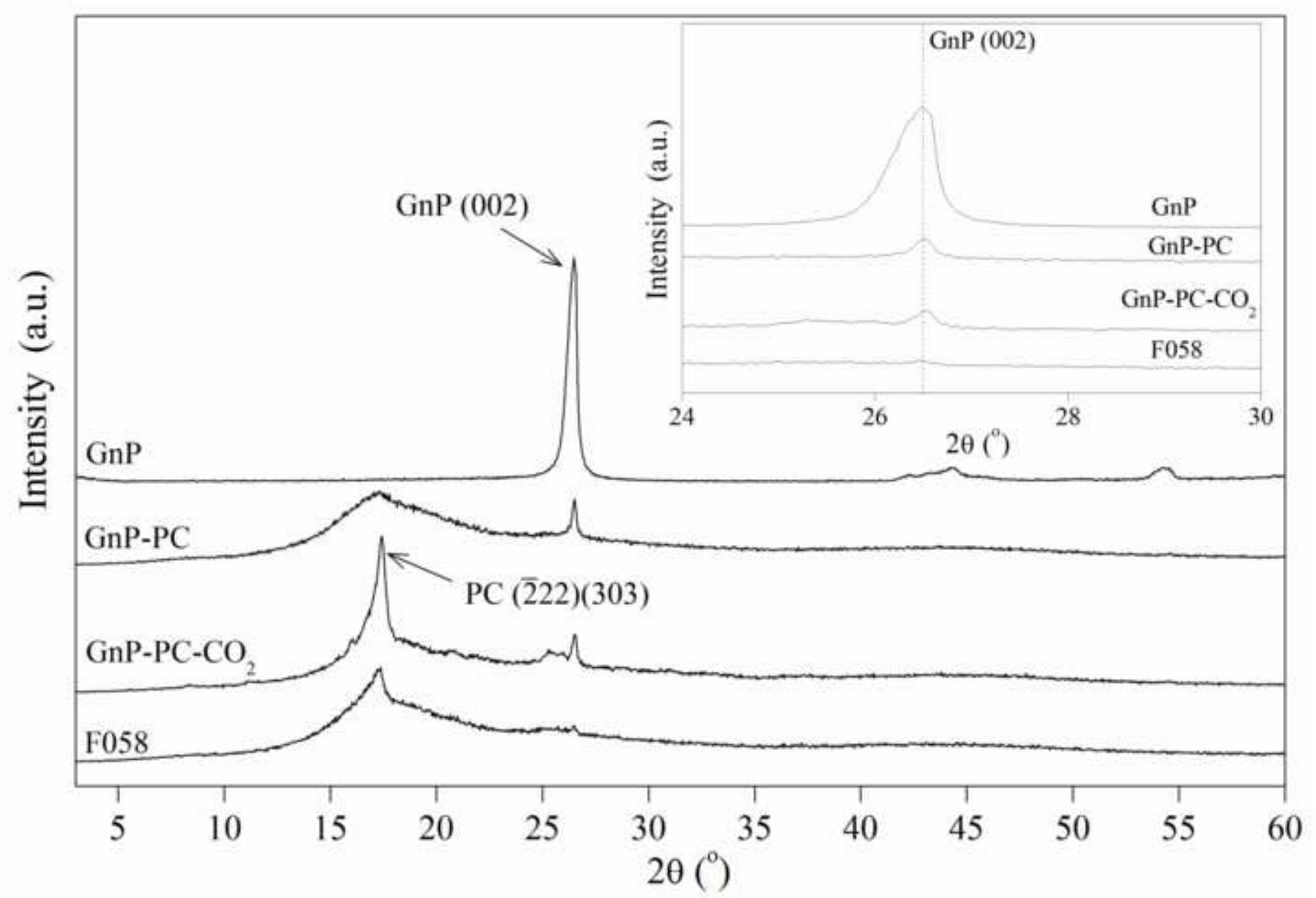




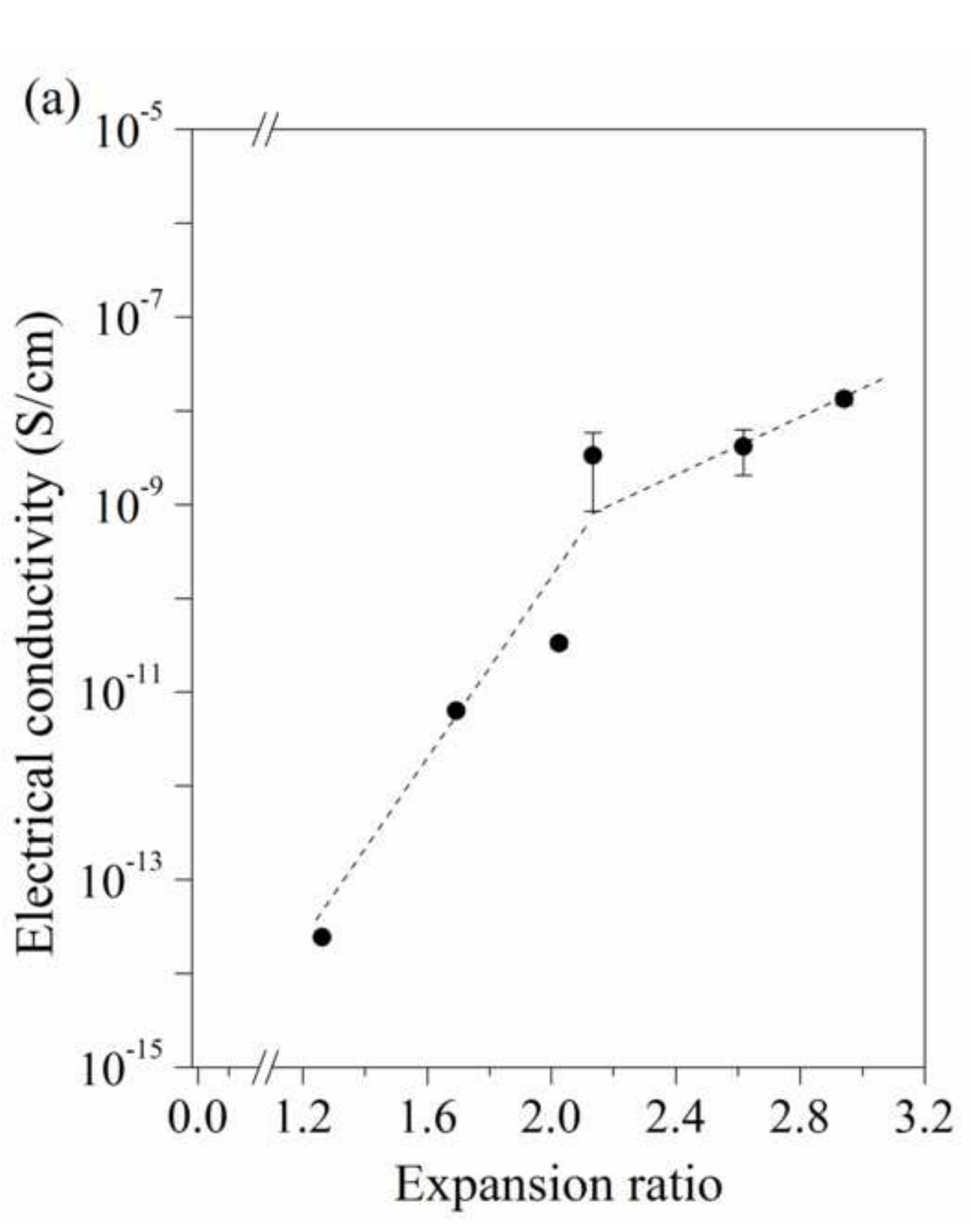




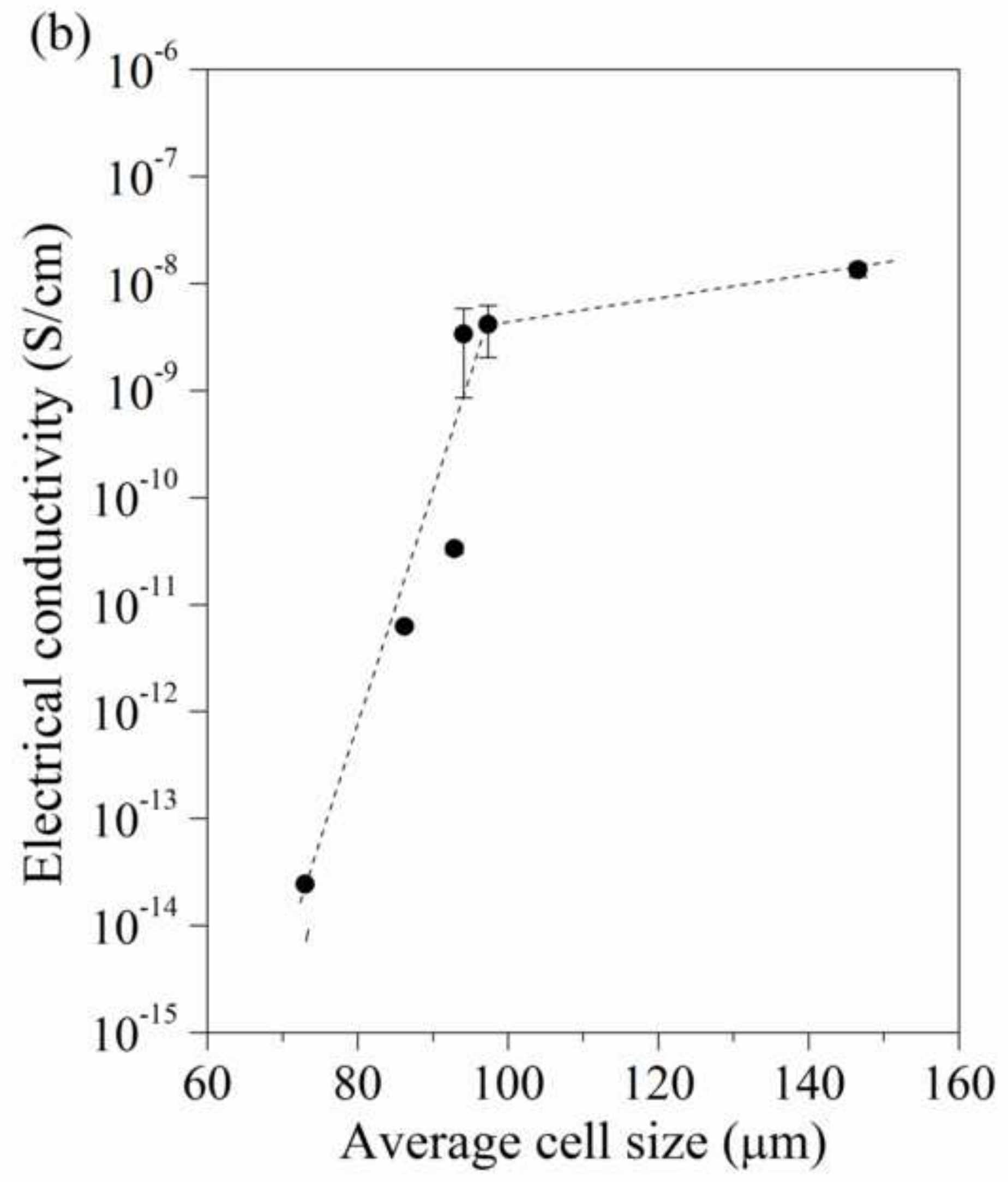






